\title{
Dificultades del profesorado para planificar, coordinar y evaluar competencias claves. Un análisis desde la Inspección de Educación
}

\section{Teachers' difficulties to plan, coordinate and evaluate key competencies. An analysis from the education inspection}

\author{
Esteban VÁZQUEZ-CANO \\ Universidad Nacional de Educación a Distancia
}

Recibido: Diciembre 2014

Evaluado: Julio 2014

Aceptado: Agosto 2014

\begin{abstract}
Resumen
Este artículo presenta una investigación en la que se analizan las dificultades del profesorado para planificar, coordinar y evaluar competencias claves en una muestra de 23 centros educativos. El tema tiene hondas repercusiones ya que una mala praxis educativa de las competencias claves puede conculcar uno de los derechos fundamentales del alumnado a ser evaluado de forma objetiva (LODE: Art.6b y RD 732/1995: Art. 13.1) y a poder superar las pruebas de evaluación consideradas necesarias para la obtención del título académico mínimo que otorga el estado español. La investigación se ha desarrollado desde una doble perspectiva metodológica. En primer lugar, es una investigación descriptiva en la que presentamos las características fundamentales de las competencias claves y la normativa básica para su desarrollo y evaluación. En segundo lugar, aplicamos un procedimiento de análisis con una doble vertiente cualitativa mediante el empleo del programa ATLAS.ti y del enfoque reticularcategorial del análisis de redes sociales con la aplicación de UCINET y el visor yED Graph Editor para abordar el análisis de las principales dificultades y obstáculos detectados. Los resultados muestran que existen serias dificultades en las tres dimensiones analizadas: "planificación", "coordinación" y "evaluación" de competencias clave. Se precisa una mayor formación del profesorado, mejorar los procesos de evaluación, desarrollar metodologías más activas y funcionales y favorecer procesos de coordinación interna en los centros educativos.
\end{abstract}

Palabas clave: competencias claves, metodología de enseñanza, evaluación, dificultades, inspección de educación.

\section{Abstract}

This article presents a research in which we analyse the difficulties of teachers to plan, coordinate, and assess key competencies in a sample of 23 schools. The issue has profound implications since a bad educational practice of key competencies may infringe one of the 
fundamental rights of students to be evaluated objectively (LODE: Art.6b and RD 732/1995: Art. 13.1) and to overcome the assessment tests deemed necessary to obtain the minimum academic degree awarded by the Spanish state. The research has been developed from a double methodological perspective. First, it is a descriptive research where we present the main characteristics of key competencies and the basic rules for its development and evaluation. Second, a qualitative analysis with a twofold approach is applied through the use of the ATLAS.ti computer program software and the reticular, category based social network analysis with UCINET and the $y E D$ Graph Editor application in order to analyse the main difficulties and obstacles. Results show that there are serious difficulties in the three analysed dimensions: "planning", "coordination" and "assessment" of key competences. More teacher training, better assessment procedures, the development of more active and functional methodologies and fostering internal coordination processes in schools, are all required measures.

Keywords: key competencies, teaching methods, assessment, difficulties, education inspection.

El desarrollo y evaluación de las competencias claves es una las principales dificultades del profesorado observadas por la inspección de educación en sus actuaciones ordinarias en los centros educativos (Autor, 2012ab). La labor inspectora de análisis y supervisión en los centros educativos tiene, como una de las principales funciones, la observación, evaluación y asesoramiento del proceso de enseñanzaaprendizaje y de los resultados académicos del alumnado (LOMCE, 2006/Art. 151). Dentro de estas funciones, el planteamiento y evaluación de las competencias claves es un aspecto crucial en el proceso de enseñanza-aprendizaje y en la evaluación del alumnado de enseñanza obligatoria (Primaria y Secundaria), ya que su superación es el elemento fundamental para otorgar la titulación mínima reconocida en el estado español (Graduado en Enseñanza Secundaria). Así se establece en el artículo 29.1 de la LOMCE (2013) en el que los alumnos/as deberán realizar una evaluación individualizada en la que se comprobará el logro de los objetivos de la etapa y el grado de adquisición de las competencias correspondientes.

El tema es tan trascendental que una mala praxis evaluativa de las competencias claves puede conculcar uno de los derechos fundamentales del alumnado a ser evaluado de forma objetiva (LODE: Art.6b y RD 732/1995: Art. 13.1) y poder superar las pruebas de evaluación consideradas necesarias para la obtención del título académico mínimo que otorga el estado español.

La planificación, coordinación y evaluación de las competencias claves no es un proceso aislado o individual del profesorado, sino una labor continua, holística y colaborativa en la que aparecen implicadas todas las materias, documentos programáticos e integrantes inmersos en el proceso educativo (Fleischman, et al., 2010; Brownell y Swaner, 2010; Autor, et al., 2011). La programación y evaluación de las competencias implica un planteamiento general que se debe materializar en un plan de centro con tres dimensiones principales: "planificación", "coordinación" y "evaluación de las competencias claves".

Este artículo analiza las principales dificultades y obstáculos del profesorado para desarrollar una correcta y eficaz planificación, coordinación y evaluación de las competencias claves en una muestra de centros educativos de las etapas de Primaria y 
Secundaria en la provincia de Toledo. El análisis y resultados obtenidos puede proporcionar pautas de actuación y recomendaciones, tanto a profesorado como equipos directivos e inspectores de educación, para mejorar los procesos de supervisión, planificación, coordinación, programación y evaluación de las competencias claves; un aspecto determinante para la obtención de la titulación académica mínima otorgada por el estado español a sus ciudadanos.

\section{El planteamiento de las competencias claves dentro del marco normativo europeo y estatal}

Las competencias claves se definen como una combinación de conocimientos, capacidades y actitudes adecuadas al contexto. Las competencias clave son aquéllas que todas las personas precisan para su realización y desarrollo personales, así como para la ciudadanía activa, la inclusión social y el empleo (Diario Oficial de la Unión Europea L 394 de 30 de diciembre de 2006). El marco de referencia europeo establece ocho competencias clave: 1. comunicación en la lengua materna; 2. comunicación en lenguas extranjeras; 3 . competencia matemática y competencias básicas en ciencia y tecnología; 4. competencia digital; 5 . aprender a aprender; 6 . competencias sociales y cívicas; 7. sentido de la iniciativa y espíritu de empresa, y 8. conciencia y expresión culturales. Además su fundamentación pedagógica y didáctica ya ha sido puesta de relieve en el contexto nacional e internacional con estudios de referencia en la materia (OCDE, 2006; European Commission, 2007; Bolívar, 2008; Escamilla, 2008; GimenoSacristán, 2008; Casanova, 2012, Proyecto Atlántida, 2014).

Las actual ley educativa (LOMCE, 2013, Preámbulo) considera que "las habilidades cognitivas, siendo imprescindibles, no son suficientes; es necesario adquirir desde edades tempranas competencias transversales, como el pensamiento crítico, la gestión de la diversidad, la creatividad o la capacidad de comunicar, y actitudes clave como la confianza individual, el entusiasmo, la constancia y la aceptación del cambio". Su materialización en el currículo básico actual (Real Decreto 126/2014) se ha realizado atendiendo a su concreción en siete competencias clave: 1. Comunicación lingüística; 2. competencia matemática y competencias básicas en ciencia y tecnología; 3 . competencia digital; 4. aprender a aprender; 5. competencias sociales y cívicas; 6 . sentido de iniciativa y espíritu emprendedor, y 7. Conciencia y expresiones culturales. Asimismo, se considera que para que exista una adquisición eficaz de las competencias y su integración efectiva en el currículo, deberán diseñarse actividades de aprendizaje integradas e interdisciplinares que permitan al alumnado avanzar hacia los resultados de aprendizaje de más de una competencia al mismo tiempo.

En esta línea de desarrollo de competencias clave se enmarca el desarrollo de las pruebas internacionales (PISA: Programme for International Student Assessment) que tratan de contribuir a la evaluación de lo que los jóvenes de 65 países saben y son capaces de hacer a los 15 años $($ OECD, 2009, 2011). La evaluación del rendimiento del estudiante español en MEC (2013) arroja unos resultados para España que ponen de relieve el nivel insuficiente obtenido en comprensión lectora, competencia matemática 
y competencia científica, muy alejado del promedio de los países de la OCDE (Tabla $1)$.

\begin{tabular}{|c|c|c|c|c|c|c|}
\hline & & 2000 & 2003 & 2006 & 2009 & 2012 \\
\hline \multirow{3}{*}{ España } & Matemáticas & & 485 & & & 484 \\
\hline & Lectura & 493 & & & 481 & 488 \\
\hline & Ciencias & & & 488 & & 496 \\
\hline \multirow{3}{*}{ OCDE } & Matemáticas & & 500 & & & 494 \\
\hline & Lectura & 500 & & & 494 & 497 \\
\hline & Ciencias & & & 498 & & 501 \\
\hline \multirow{3}{*}{$\begin{array}{l}\text { Diferencia } \\
\text { Promedio } \\
\text { OCDE/España }\end{array}$} & Matemáticas & & 15 & & & 10 \\
\hline & Lectura & 7 & & & 13 & 9 \\
\hline & Ciencias & & & 10 & & 5 \\
\hline
\end{tabular}

Tabla 1. Evolución de los resultados globales PISA. (Fuente MEC, 2013)

Hay que tener en cuenta que el objetivo de las pruebas internacionales PISA es medir el nivel de competencias y no de contenidos teóricos. Por lo tanto, la tendencia actual es hacia una simplificación del currículo, de acuerdo con las directrices de la Unión Europea (Diario Oficial de la Unión Europea, 2006), para así poder proporcionar un conocimiento sólido de los contenidos de forma práctica y aplicada que garantice la efectividad en la adquisición de las competencias claves.

El planteamiento de estas competencias claves en la LOE (2006) (parcialmente en vigor para el curso 2015/16) y en la normativa actual (LOMCE, 2013) se realiza desde dos áreas prioritarias: los principios pedagógicos y la evaluación. El artículo 6.2.b de la (LOMCE, 2013) establece que "las competencias con aquellas capacidades necesarias para aplicar de forma integrada los contenidos propios de cada enseñanza y etapa educativa, con el fin de lograr la realización adecuada de actividades y la resolución eficaz de problemas complejos". Asimismo, los artículos 20.2 y 28.2 (LOMCE, 2013) establecen que los alumnos/as de primaria y secundaria promocionarán de curso y etapa atendiendo al logro de los objetivos y al grado de adquisición de las competencias correspondientes. Cada una de las áreas contribuye al desarrollo de diferentes competencias y, a su vez, cada una de las competencias claves se alcanzará como consecuencia del trabajo en varias áreas o materias. El trabajo en las áreas y materias del currículo para contribuir al desarrollo de las competencias básicas debe complementarse con diversas medidas organizativas y funcionales, imprescindibles para su desarrollo. Así, la organización y el funcionamiento de los centros y las aulas, la participación del alumnado, las normas de régimen interno, el uso de determinadas metodologías y recursos didácticos, o la concepción, organización y funcionamiento de la biblioteca escolar, entre otros aspectos, pueden favorecer o dificultar el desarrollo de competencias asociadas a la comunicación, el análisis del entorno físico, la creación, la convivencia y la ciudadanía, o la alfabetización digital (Sanmartí, 2007; VázquezCano, 2012). Igualmente, la acción tutorial permanente puede contribuir de modo determinante a la adquisición de competencias relacionadas con la regulación de los aprendizajes, el desarrollo emocional o las habilidades sociales. 


\section{Objetivos}

Partimos de la siguiente premisa: "La actuación de la inspección de educación observa de forma sistemática dificultades del profesorado para abordar la planificación, coordinación, desarrollo y evaluación de las competencias claves del alumnado". Esta apreciación debe ser refrendada por un estudio sistemático longitudinal en diferentes contextos socioeducativos y tipologías de centros que puedan establecer los obstáculos y dificultades más comunes y difíciles de sistematizar por parte del profesorado y equipos directivos. Este planteamiento se materializa en los siguientes objetivos:

- Analizar las dificultades en los procesos de planificación de centro para abordar la evaluación de las competencias claves.

- Conocer las dificultades en los procesos de coordinación didáctica y organizativa que se establecen en los centros educativos para el desarrollo y evaluación de las competencias claves.

- Establecer las dificultades y obstáculos más significativos del profesorado en la programación y evaluación de las competencias claves.

A partir de estas cuestiones, planteamos tres dimensiones específicas que servirán de referencia en el desarrollo de la metodología de investigación: planificación, coordinación, metodología y evaluación de las competencias claves.

\section{Metodología}

La fase de recogida de información estuvo inserta en los procesos y funciones normalizados en la inspección educativa. Este modelo se basa en el principio de intervención y supervisión de la inspección de los procesos de evaluación por medio de la visita al centro (Daresh y Playko, 1995), asistencia a las reuniones de los órganos colegiados, observación de prácticas metodológicas y evaluadoras en el aula y el análisis de documentos programáticos y de práctica docente: programaciones y unidades didácticas, diarios de clase del profesorado, etc. (Dean, 1997; Strauss y Corbin, 1997; Clandinin y Connelly, 2000; Charmaz, 2001; Casanova, 2004).

Castilla-La Mancha es una comunidad autónoma que cuenta con 1511 centros educativos. Para la muestra se seleccionaron centros pertenecientes a la actuación prioritaria de la Inspección de Educación para la zona de Talavera de la Reina en Toledo. El total de centros participantes en la muestra fue de 23 (12 centros de enseñanza secundaria ubicados 8 de ellos en núcleos urbanos de más de 20.000 habitantes y 4 en zona rural por debajo de 5.000 habitantes y 11 de educación primaria 6 de ellos en zona urbana y 5 en zona rural) y una muestra total participante de 734 personas correspondientes a los cuatro perfiles fundamentales de la comunidad educativa: profesorado, equipos directivos, padres, madres y alumnado. El proceso de recogida de datos tuvo lugar durante los cursos académicos comprendidos entre los años 2010/2013 y el proceso de análisis durante el curso 2013/14. En la Tabla 2, se puede ver la muestra de participantes en la investigación. 


\begin{tabular}{|c|c|c|}
\hline $\begin{array}{c}\text { Comunidad educativa } \\
\text { participante }\end{array}$ & N. ${ }^{\circ}$ & $\begin{array}{c}\text { Porcentaje de } \\
\text { casos }\end{array}$ \\
\hline Profesorado & 523 & $71.25 \%$ \\
\hline Equipos directivos & 45 & $6,13 \%$ \\
\hline Padres y madres & 101 & $13.76 \%$ \\
\hline Alumnado & 65 & $8,85 \%$ \\
\hline Total & 734 & $100 \%$ \\
\hline
\end{tabular}

Tabla 2. Comunidad educativa participante.

Las fuentes de información empleadas y el procedimiento analítico se muestran en la Tabla 3:

\begin{tabular}{lcl}
\hline \multicolumn{1}{c}{ Fuentes de información } & Análisis & \multicolumn{1}{c}{ Tratamiento } \\
\hline $\begin{array}{l}\text { 1. Actas de las sesiones de } \\
\text { evaluación y observación } \\
\text { participante en las sesiones de } \\
\text { evaluación. }\end{array}$ & $\begin{array}{l}\text { Cualitativo/ } \\
\text { Cuantitativo }\end{array}$ & $\begin{array}{l}\text { Word cruncher: estadística de conceptos. } \\
\text { Memos: anotaciones en observación } \\
\text { participante. }\end{array}$ \\
\hline $\begin{array}{l}\text { 2. Entrevista a profesores, } \\
\text { tutores, jefes/as de } \\
\text { departamento, alumnos, } \\
\text { familias y equipo directivo. }\end{array}$ & Cualitativo & $\begin{array}{l}\text { Codes Primary-Documents Table: Códigos } \\
\text { primarios. Networks: redes de relaciones entre } \\
\text { conceptos primarios y códigos de análisis. } \\
\text { Hierarchy: jerarquías de conceptos y } \\
\text { dificultades. }\end{array}$ \\
\hline $\begin{array}{l}\text { 3. Grupo de discusión con } \\
\text { profesores, tutores, jefes/as de } \\
\text { departamento, alumnos, } \\
\text { familias y equipo directivo. }\end{array}$ & Reticular & $\begin{array}{l}\text { Grabación y Transcripción. Análisis de redes } \\
\text { sociales y representación por grafo. }\end{array}$ \\
\hline $\begin{array}{l}\text { 4. Documentos programáticos } \\
\text { de los centros educativos. }\end{array}$ & Cualitativo/ & $\begin{array}{l}\text { Word cruncher: estadística de conceptos. } \\
\text { Codes Primary-Documents Table: Códigos } \\
\text { primarios. }\end{array}$ \\
\hline $\begin{array}{l}\text { 5. Instrumentos de evaluación } \\
\text { del profesorado. }\end{array}$ & Cualitativo & $\begin{array}{l}\text { Memos: anotaciones en observación } \\
\text { participante. Networks: redes de relaciones } \\
\text { entre conceptos primarios y códigos de } \\
\text { análisis. }\end{array}$ \\
\hline $\begin{array}{l}\text { Hierarchy: jerarquías de conceptos y } \\
\text { dificultades. }\end{array}$
\end{tabular}

Tabla 3. Fuentes de información y tratamiento analítico.

Los datos obtenidos se estructuraron en dos fases de análisis. En primer lugar, recurrimos a un análisis cualitativo mediante identificación de frecuencias textuales más recurrentes en las fuentes de información 1, 2, 4 y 5. Para ello, hemos empleado la herramienta de citas de texto, memos y "word-cruncher" del programa Atlas-Ti 7.0. En segundo lugar, se analizaron los grupos de discusión (Fuente de información, 3) desde la perspectiva del Análisis de Redes Sociales (ARS) cuya metodología nos permite adentrarnos en la interacción de las conversaciones desde la selección de bloques temáticos y sus relaciones conceptuales y semánticas.

Este procedimiento de análisis de las intervenciones en grupos de discusión nos permite entender la forma y estructura de esas relaciones como un todo y esto es 
considerado clave para lograr entender los mecanismos causales de la dificultad que entraña el tratamiento de las competencias claves; permitiéndonos descubrir pautas de interacción ocultas (Barabási, 2002; Knoke y Yang, 2008). El ARS se fundamenta en la idea de que las estructuras de relaciones entre elementos explican mejor el conjunto, el entorno social y también a cada uno de los elementos, que los atributos de estos tomados unitariamente (Castells y Monge, 2011; Caverlee, Liu y Webb, 2010).

Por lo tanto, el procedimiento de análisis conjunto, cualitativo y reticular de las fuentes de información escritas y los grupos de discusión, nos permite obtener una información más completa que integre variables latentes no siempre identificables mediante otros análisis meramente cuantitativos. Para el procedimiento de análisis, hemos empleado tres programas informáticos: el programa Atlas-Ti 7.0 para el tratamiento de las fuentes de información escrita (programaciones didácticas, actas, etc.), el programa UCINET 6 para el análisis reticular de la interacción comunicativa en los grupos de discusión y el visor yED Graph Editor 3.11.1 para editar el grafo resultante de la matriz de la red obtenida con UCINET y así poderlo hacer más comprensible. El esquema matricial que se ha empleado para la generación del grafo ha sido el siguiente:

$$
\operatorname{Pr}(\mathbf{Y}=\mathbf{y})=\left(\frac{1}{\kappa}\right) \exp \left\{\sum_{A} \eta_{A} g_{A}(\mathbf{y})\right\}
$$

Donde $\eta \mathrm{A}$ es el parámetro correspondiente a la configuración A (cuyo resultado no podrá ser cero si todos los pares de variables en A se asumen como "condicionalmente dependientes"). Asimismo, gA(y) = yijIIAyij es la estadística de configuración de red que corresponde a $\mathrm{A} ; \mathrm{gA}(\mathrm{y})=1$ si coincide con la observación de "y" en la red, y si no parece en la red su resultado es $0 . \kappa$ es una cantidad que asegura que la normalización (1) es una adecuada distribución de probabilidad. Todos los modelos de grafos aleatorios exponenciales adoptan la forma Eq. (1) lo que implica una distribución de probabilidad general de los gráficos en " $n$ " nodos teniendo en cuenta que existen diferentes supuestos de dependencia que tienen la consecuencia de escoger diferentes tipos de configuraciones como relevantes para el modelo. Tomando en consideración esta ecuación, las únicas configuraciones que son relevantes para el modelo son aquellas en los que todos los lazos posibles en la configuración son mutuamente contingentes entre sí.

Este tipo de metodología nos proporciona un paradigma de investigación más sólido que el meramente cualitativo al explicar con más detalle procesos internos de centro educativo y externos en la relación entre todos los agentes intervinientes en la comunidad educativa. Por consiguiente, nuestra metodología se inscribe en el marco de las corrientes epistemológicas interpretativas y dialógicas de análisis de contenido y análisis de redes con representaciones matriciales y con grafos. 


\section{Resultados y discusión}

La primera fase consistió en el análisis por parte de dos investigadores independientes de la base de datos textual recogida conforme a las fuentes de información escritas de la investigación. Se estableció un primer análisis de las unidades textuales para medir la tendencia de intercódigos y solapamientos conceptuales. Para ello, se calculó el índice Guetzkow's U con el que se mide la fiabilidad del número de unidades identificadas por dos investigadores independientes, conforme a la siguiente fórmula (Holsti 1969):

$$
\mathrm{U}=(\mathrm{O} 1-\mathrm{O} 2) /(\mathrm{O} 1+\mathrm{O} 2)
$$

$\mathrm{O} 1$ y $\mathrm{O} 2$ representan el número de unidades categorizadas respectivamente por cada uno de los investigadores que realizaron la codificación preliminar. Esta codificación supuso la identificación de 3332 unidades de significado agrupadas en 52 categorías deductivo-inductivas. Este análisis preliminar presentó un resultado en el índice Guetzkow's U de .0198 , lo que representa casi un $90 \%$ de coincidencia en el número de unidades identificadas por ambos investigadores. Asimismo, para verificar la consistencia interna de las unidades textuales identificadas se empleó el cotejo comparado de las unidades codificadas en el programa "excel". La consistencia fue alta con un $84,45 \%$ de coincidencia en el primer análisis, lo que se considera un índice alto (Simons, 1993; Weingart, Olekalns y Smith, 2004). Posteriormente, en una segunda fase después de un proceso de comparación intercategorías se identificaron 3 dominios con 17 núcleos temáticos (macrocategorías) en torno a los cuales se han agrupado todas las unidades de significado y se procedió a establecer el índice Kappa de Cohen para analizar la fiabilidad intercategorial. Para ello, empleamos el modelo sugerido por Brennan y Prediger (1981) calculado conforme a la siguiente fórmula:

$$
\kappa=\left(\Sigma \mathrm{P}_{\mathrm{ii}}-\Sigma \mathrm{P}_{\mathrm{i}} \times \mathrm{P}_{\mathrm{i}}\right) /\left(1-\Sigma \mathrm{P}_{\mathrm{i}} \times \mathrm{P}_{\mathrm{i}}\right)
$$

Donde $\Sigma$ Pii es el sumatorio de observaciones comunes y $\Sigma$ Pi x Pi refleja la probabilidad de coincidencia (Holsti 1969). Para determinar la coincidencia y fiabilidad de los conceptos asignados, se procedió a comparar el análisis preliminar con el desarrollo de un matriz de consistencia intercódigos aplicada a las 17 categorías y subniveles. La Tabla 4 muestra la matriz resultante. 


\begin{tabular}{|c|c|c|c|c|c|c|c|c|c|c|c|c|c|c|c|c|c|}
\hline Cod.1/2 & pe & $\mathrm{py}$ & ex & ie & at & pa & co & cp & ri & cb. & ip & ed & ren & ac & ec & $\mathrm{pf}$ & ut \\
\hline pe & 173 & 57 & 31 & 33 & 19 & 16 & 23 & 13 & 31 & 13 & 17 & 31 & 54 & 67 & 19 & 17 & 43 \\
\hline py & 33 & 54 & 41 & 42 & 18 & 15 & 12 & 21 & 54 & 21 & 18 & 10 & 23 & 62 & 18 & 18 & 41 \\
\hline $\mathrm{ex}$ & 37 & 61 & 187 & 50 & 31 & 21 & 17 & 31 & 23 & 31 & 32 & 87 & 21 & 45 & 24 & 32 & 32 \\
\hline ie & 43 & 36 & 45 & 90 & 32 & 16 & 18 & 32 & 54 & 32 & 25 & 34 & 67 & 31 & 25 & 25 & 39 \\
\hline at & 18 & 47 & 78 & 13 & 100 & 27 & 19 & 12 & 67 & 12 & 27 & 31 & 54 & 67 & 19 & 17 & 43 \\
\hline pa. & 34 & 31 & 65 & 21 & 41 & 101 & 21 & 17 & 54 & 17 & 28 & 10 & 23 & 62 & 18 & 18 & 41 \\
\hline$s$ & 52 & 54 & 43 & 31 & 23 & 23 & 92 & 13 & 21 & 13 & 23 & 62 & 18 & 18 & 41 & 32 & 62 \\
\hline cp & 65 & 23 & 23 & 32 & 45 & 21 & 21 & 134 & 32 & 13 & 49 & 67 & 42 & 17 & 15 & 31 & 43 \\
\hline ri & 71 & 54 & 21 & 12 & 23 & 34 & 21 & 18 & 101 & 21 & 21 & 65 & 23 & 23 & 32 & 45 & 21 \\
\hline cb & 49 & 67 & 42 & 17 & 15 & 31 & 43 & 21 & 12 & 231 & 35 & 31 & 54 & 67 & 19 & 17 & 13 \\
\hline ip & 31 & 54 & 67 & 19 & 17 & 43 & 41 & 67 & 11 & 43 & 89 & 32 & 45 & 34 & 23 & 21 & 21 \\
\hline ed & 10 & 23 & 62 & 18 & 18 & 41 & 32 & 62 & 54 & 23 & 21 & 299 & 41 & 21 & 21 & 31 & 31 \\
\hline re & 87 & 21 & 45 & 24 & 32 & 32 & 39 & 45 & 23 & 21 & 31 & 32 & 200 & 32 & 53 & 32 & 32 \\
\hline ac & 34 & 67 & 31 & 25 & 25 & 39 & 43 & 31 & 21 & 42 & 32 & 11 & 32 & 378 & 34 & 12 & 12 \\
\hline ec & 65 & 89 & 98 & 26 & 27 & 37 & 41 & 67 & 67 & 21 & 12 & 13 & 21 & 23 & 365 & 17 & 17 \\
\hline pf & 78 & 76 & 51 & 12 & 28 & 41 & 43 & 67 & 54 & 17 & 17 & 25 & 25 & 39 & 13 & 460 & 13 \\
\hline ut & 88 & 34 & 31 & 19 & 31 & 32 & 41 & 62 & 23 & 12 & 19 & 21 & 17 & 11 & 15 & 29 & 278 \\
\hline Total & 968 & 848 & 961 & 484 & 525 & 570 & 567 & 713 & 702 & 583 & 496 & 861 & 760 & 997 & 754 & 854 & 782 \\
\hline Prom. & 569 & 94.2 & 56,5 & 28.4 & 58,3 & 63,3 & 63 & 79,2 & 78 & \begin{tabular}{|l|}
64,7 \\
\end{tabular} & 29.1 & \begin{tabular}{|l|}
95,6 \\
\end{tabular} & 84.4 & 58.6 & \begin{tabular}{|l|}
44,3 \\
\end{tabular} & \begin{tabular}{|l|}
50,2 \\
\end{tabular} & 46 \\
\hline \multicolumn{18}{|c|}{$\begin{array}{l}\text { 3332 Unidades de significado. Categorizadas conforme a las } 17 \text { categorias siguientes: } \\
\text { Proyecto Didáctico (pe); Plan de evolución (pv); Procesos de evaluación (ev); Informe de evaluación (ie); Plan de acción tutorial (at); Planificación } \\
\text { de actividades complementarias y extraescolares (pa); Coordinación y análisis por parte de los órganos colegiados (co); Coordinación y analisis po } \\
\text { parte de los equipos de ciclo y dpto. didácticos (cp); Relaciones interdepartamentales e interdisciplinares (ri); Coordinación de competencias a travé } \\
\text { de la Biblioteca (cb); Integración de competencias en proyectos (ip); Estrategias didácticas (ed); Relación contenidos y competencias (rc); Anotación } \\
\text { del progreso de las competencias (ac); Evaluación y calificación de las competencias (ec); Planes de formación (pf); Uso de las TIC como ej } \\
\text { transversal (ut). }\end{array}$} \\
\hline
\end{tabular}

Tabla 4. Resultados de la consistencia de la matriz intercódigos.

Los resultados muestran un índice Kappa de Cohen con alta fiabilidad y consistencia $\kappa=86.56$. Los valores por encima de .80 son considerados en la literatura científica como resultados muy confiables y, en este caso, superior a estudios similares (Brett et al., 1998; Lombard, Snyder-Duch y Bracken, 2002). Los resultados finales de unidades conceptuales analizadas agrupadas por dominios se pueden ver en la Tabla 5.

\begin{tabular}{|c|c|c|c|}
\hline Dominios & Macrocategorías & $\begin{array}{c}\text { Uni } \\
\text { sig }\end{array}$ & $\begin{array}{l}\text { les de } \\
\text { cado }\end{array}$ \\
\hline \multirow{6}{*}{ Planificación } & 1. Proyecto Didáctico. & 173 & 5,19 \\
\hline & 2. Plan de evolución. & 54 & 1,62 \\
\hline & 3. Procesos de evaluacion. & 187 & 5,61 \\
\hline & 4. Informe de evaluación. & 90 & 2,70 \\
\hline & 5. Plan de acción tutorial. & 100 & 3,00 \\
\hline & $\begin{array}{l}\text { 6. Planificación de actividades complementarias y } \\
\text { extraescolares. }\end{array}$ & 101 & 3,03 \\
\hline \multirow{5}{*}{ Coordinación } & $\begin{array}{l}\text { 7. Coordinación y análisis por parte de los órganos } \\
\text { colegiados. }\end{array}$ & 92 & 2,76 \\
\hline & $\begin{array}{l}\text { 8. Coordinación y análisis por parte de los equipos de ciclo y } \\
\text { dpto. didácticos. }\end{array}$ & 134 & 4,02 \\
\hline & 9. Relaciones interdepartamentales e interdisciplinares. & 101 & 3,03 \\
\hline & 10. Coordinación de competencias a través de la Biblioteca. & 231 & 6,93 \\
\hline & 11. Integración de competencias en proyectos. & 89 & 2,67 \\
\hline
\end{tabular}




\begin{tabular}{rlcc}
\hline & 12. Estrategias didácticas. & 299 & 8,97 \\
\cline { 2 - 5 } Didáctica y & 13. Relación contenidos y competencias. & 200 & 6,00 \\
\cline { 2 - 5 } evaluación & 14. Anotación del progreso de las competencias. & 378 & 11,34 \\
\cline { 2 - 5 } & 15. Evaluación y calificación de las competencias. & 365 & 10,95 \\
\cline { 2 - 5 } & 16. Planes de formación. & 460 & 13,80 \\
\cline { 2 - 5 } & 17. Uso de las TIC como eje transversal. & 278 & 8,34 \\
\hline Total & & 3332 & $100 \%$ \\
\hline
\end{tabular}

Tabla 5. Dominios y porcentajes de significado.

La descripción de los resultados cualitativos y estadísticos se muestran atendiendo a las dimensiones planteadas en la investigación y se representan con las herramientas de análisis prioritarias en el tratamiento de datos obtenido en Atlas-Ti (Tabla II).

\section{Dimensión 1: Planificación de las competencias claves en el centro educativo}

Uno de los procesos fundamentales para un correcto desarrollo de las competencias claves y su posterior evaluación es el poder disponer en el centro educativo de un plan interno integrado en sus documentos programáticos (Proyecto Educativo de CentroPEC y Programación Genera Anual-PGA) para que el profesorado planifique también su labor docente y programática conforme a estos principios generales. En esta dimensión, analizamos cómo se concretan los procesos de planificación de las competencias claves de los centros educativos, de forma previa a su materialización en las programaciones didácticas de los departamentos o equipos de ciclo.

Con ello, pretendemos comprobar el diseño pedagógico de centro como elemento favorecedor de los procesos de programación y evaluación de las competencias por parte del profesorado; aspecto esencial para un desarrollo interdisciplinar y colaborativo de las mismas. En la Tabla 6, se presentan las frecuencias de significado de las categorías más determinantes para entender las dificultades en el proceso planificador de las competencias claves.

\begin{tabular}{|c|c|c|c|c|}
\hline $\begin{array}{l}\text { Dominio: Dificultad Planificación de } \\
\text { Competencias }\end{array}$ & $\begin{array}{c}\text { Casos/ } \\
\text { Citas }\end{array}$ & $\%$ Casos & Nb Words & \% Words \\
\hline Informe a familias. & 101 & $12,9 \%$ & 432 & $19,8 \%$ \\
\hline Plan de centro. & 135 & $17,3 \%$ & 378 & $17,3 \%$ \\
\hline Plan didáctico. & 137 & $17,6 \%$ & 354 & $16,2 \%$ \\
\hline Procesos de evaluación. & 81 & $10,4 \%$ & 199 & $9,1 \%$ \\
\hline Plan de acción tutorial. & 134 & $17,2 \%$ & 432 & $19,8 \%$ \\
\hline Planificación de actv. compl. y extraescolares. & 189 & $24,3 \%$ & 378 & $17,3 \%$ \\
\hline Total & 777 & 100 & 2173 & 100 \\
\hline
\end{tabular}

Tabla 6. Dificultades en el proceso de planificación de competencias. 
Las unidades de significado que mayor porcentaje de aparición han tenido en la revisión de documentos y en las entrevistas realizadas han sido: "La falta de plan de centro y didáctico" (Casos=272/Nb-Words: 732), y "la dificultad para dinamizar las actividades extraescolares y el plan de acción tutorial para desarrollar competencias" (Casos $=323 / \mathrm{Nb}$-Words: 810 ).

En la Figura 1, de forma más visual, se muestra el esquema ontosemiótico de las principales dificultades en el proceso de planificación de las competencias claves en porcentajes de aparición en la unidad hermenéutica de Atlas-Ti.

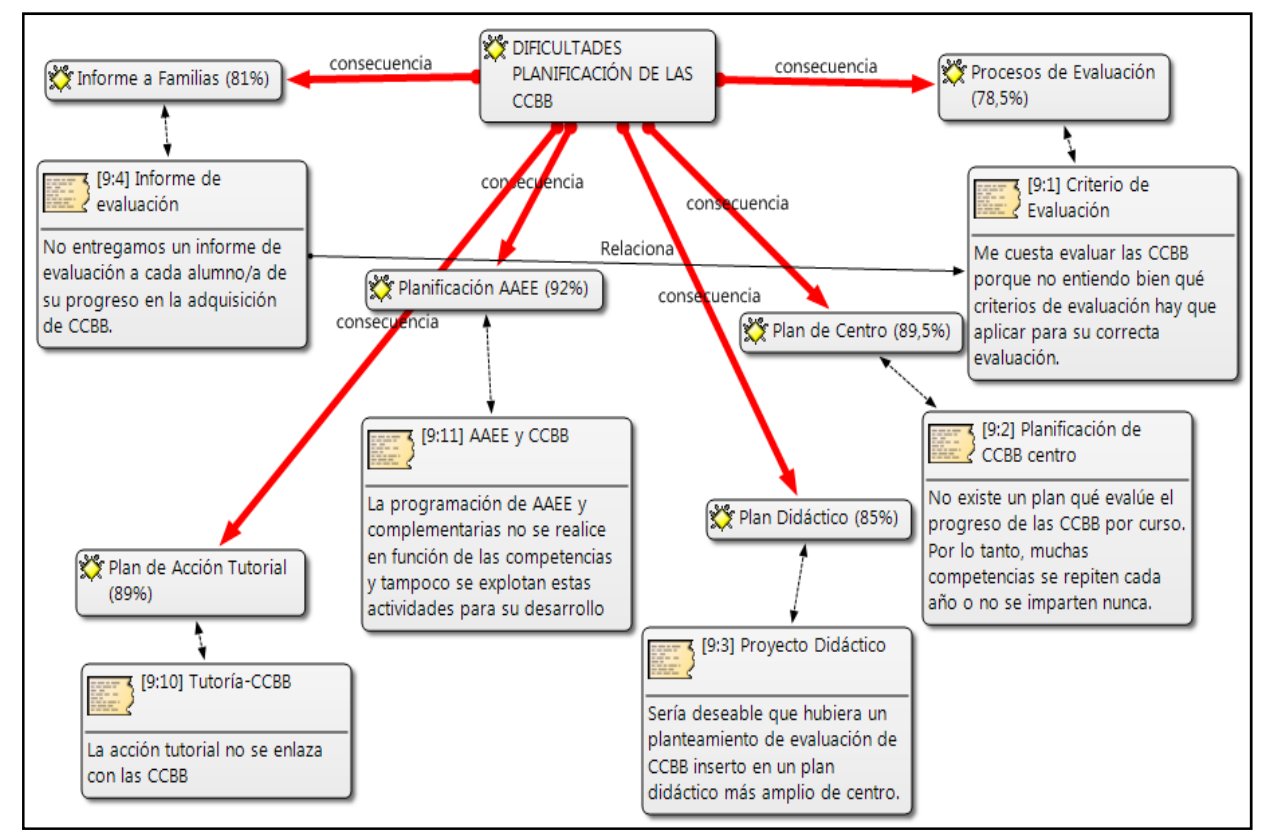

Figura 1. Dificultades en el proceso de planificación de competencias claves.

Las principales dificultades observadas en los procesos de planificación de las competencias claves en los centros educativos se concretan en los siguientes ámbitos:

- Un $86,95 \%$ de los centros educativos $\mathrm{N}=20$ no dispone de un "Plan de Centro" para contextualizar las competencias claves y orientar su desarrollo. Estos planes o documentos no aparecen insertados en los documentos programáticos principales de los centros educativos Proyecto Educativo de Centro (PEC) y Programación General Anual (PGA).

- Un $81 \%$ de los centros educativos $\mathrm{N}=19$ no dispone de un documento informativo a familias que complemente el tradicional boletín de notas en el que se informe a las familias del progreso y superación de determinados indicadores de habilidad o competencia asociados a cada una de las competencias y valorados de forma conjunta por el 
profesorado. Este aspecto correlaciona negativamente con los procesos de evaluación de las competencias.

- Un $78,5 \%$ de los profesores/as analizados $\mathrm{N}=411$ no entienden bien cómo relacionar los criterios de evaluación del currículo con la evaluación de las competencias.

- Un $89 \%$ de los centros educativos analizados $\mathrm{N}=20$ no disponen de una organización del plan de acción tutorial que ayude al desarrollo y planificación de las competencias claves (una de las recomendaciones realizadas por el propio Ministerio de Educación para favorecer el desarrollo de las competencias claves).

- Un $92 \%$ de los centros educativos analizados $\mathrm{N}=21$ no programan y aprueban un plan de actividades complementarias o extraescolares para su desarrollo durante un curso académico y establecen su relación con el desarrollo de las competencias claves de forma interdisciplinar.

El proceso de planificación de las competencias es fundamental para abordarlas dependiendo del contexto socioeducativo y académico de cada centro educativo y, como consecuencia, orientar su inserción en las programaciones didácticas y en la práctica docente en el aula. Una planificación contextualizada de las competencias acorde con las dinámicas socioeducativas de los centros educativos es esencial para su correcto desarrollo. Derivado del análisis de esta primera dimensión, podemos comprobar cómo no existen procesos fuertes y consolidados para planificar las competencias claves de forma general en los centros educativos, lo que dificulta significativamente los procesos posteriores de coordinación, programación y evaluación de las mismas.

\section{Dimensión 2: Coordinación de las competencias claves en centros educativos.}

La coordinación en el desarrollo de las competencias claves en los centros educativos se erige en una de las acciones claves para la consecución de un completo y objetivo proceso didáctico y evaluador de las mismas. El sentido que tiene coordinar el desarrollo de las competencias por niveles es poder organizar procesos metodológicos contextualizados en un plan didáctico de centro en el cual cada departamento o equipo de ciclo planifique y coordine su progreso, evaluación y criterios de promoción y titulación.

De esta forma, no se repiten actividades competenciales de forma sistemática cada curso escolar, dejando otras competencias casi sin desarrollar a lo largo de una etapa. La contribución de cada materia a cada una de las competencias no es la misma; por lo que la coordinación en actividades y en porcentajes de evaluación es un proceso necesario previo a su desarrollo en el aula. No tiene sentido que cada profesor/a imparta, desde su materia, competencias que no estén entroncadas en planes coordinados, - primero de forma general en el centro educativo- y posteriormente en 
su departamento didáctico, para finalmente ser puestos en común con la participación de todo el profesorado adscrito a los departamentos didácticos o equipos de ciclo.

En la Tabla 7, se presentan las frecuencias de significado de las categorías más determinantes para entender las dificultades de coordinación didáctica de las competencias claves.

\begin{tabular}{|c|c|c|c|c|}
\hline Dominio: Dificultad Coordinación de Ccompetencias & Casos & $\begin{array}{c}\% \\
\text { Casos }\end{array}$ & $\begin{array}{c}\text { Nb } \\
\text { Words }\end{array}$ & $\begin{array}{c}\% \\
\text { Words }\end{array}$ \\
\hline Coordinación y análisis por parte de los órganos colegiados. & 87 & $11,4 \%$ & 199 & $11,4 \%$ \\
\hline Coordinación y análisis equipos de ciclo y dpto. didácticos. & 155 & $20,4 \%$ & 388 & $22,2 \%$ \\
\hline Relaciones interdepartamentales e interdisciplinares. & 181 & $23,8 \%$ & 415 & $23,8 \%$ \\
\hline Coordinación de competencias a través de la Biblioteca. & 191 & $25,1 \%$ & 437 & $25,1 \%$ \\
\hline Integración de competencias en proyectos. & 144 & $18,9 \%$ & 301 & $17,2 \%$ \\
\hline Total & 758 & 100 & 1740 & 100 \\
\hline
\end{tabular}

Tabla 7. Dificultades en el proceso de coordinación de competencias.

Las frecuencias más determinantes que explican la dificultad para coordinar procesos didácticos y de evaluación de competencias claves están determinadas principalmente por la "falta de coordinación y análisis por parte de los órganos colegiados y de los equipos de ciclo y departamentos didácticos" (Casos=242/NbWords: 587). También es altamente significativa la dificultad a la hora de establecer relaciones interdepartamentales e interdisciplinares que fructifiquen en proyectos de centro $(\operatorname{Casos}=325 / \mathrm{Nb}$-Words: 716$)$. Asimismo, no hay proyectos de lectura o de dinamización de la biblioteca para el fomento, de forma coordinada, de las competencias (Casos=191/Nb-Words: 437).

En la Figura 2, de forma más visual, se muestran las principales dificultades en el proceso de coordinación de las competencias claves en porcentajes de aparición en la unidad hermenéutica de Atlas-Ti y las correlaciones entre categorías.

Las principales dificultades observadas en los procesos de coordinación de las competencias claves en los centros educativos se concretan en los siguientes ámbitos:

- Un $51 \%$ de los centros educativos $\mathrm{N}=12$ no realiza reuniones de coordinación o análisis del progreso de adquisición de las competencias claves por parte de los órganos colegiados (Claustro, Consejo escolar y Comisión de Coordinación Pedagógica). Esto supone un considerable obstáculo para mejorar los procesos de coordinación de las competencias.

- Un $87 \%$ del profesorado $\mathrm{N}=455$ no se coordina en su departamento didáctico o equipo de ciclo para unificar criterios a la hora de programar y evaluar las competencias claves $\mathrm{y}$, de esta manera, favorecer una evaluación consensuada y equitativa entre los diferentes cursos y niveles.

- Un $91 \%$ del profesorado $\mathrm{N}=476$ no participa en reuniones de coordinación que favorezcan el desarrollo de las competencias de forma interdepartamental e 
interdisciplinar con otros compañeros/as y materias afines para coordinar contenidos comunes de trabajo.

- Un $87 \%$ del profesorado $\mathrm{N}=455$ no realiza proyectos de centro en los que involucrar a todos los integrantes de la comunidad educativa en el desarrollo conjunto de las competencias claves y potenciar su aplicabilidad y significatividad en el contexto socioeducativo.

- Un $95 \%$ de los centros $\mathrm{N}=22$ no dispone de un plan para la dinamización de la biblioteca del centro enfocado al desarrollo de las competencias claves.

- Un $91 \%$ de los centros $\mathrm{N}=21$ no realiza el seguimiento del desarrollo, evaluación y adquisición de las competencias claves y no refleja acuerdos de mejora en sus actas.

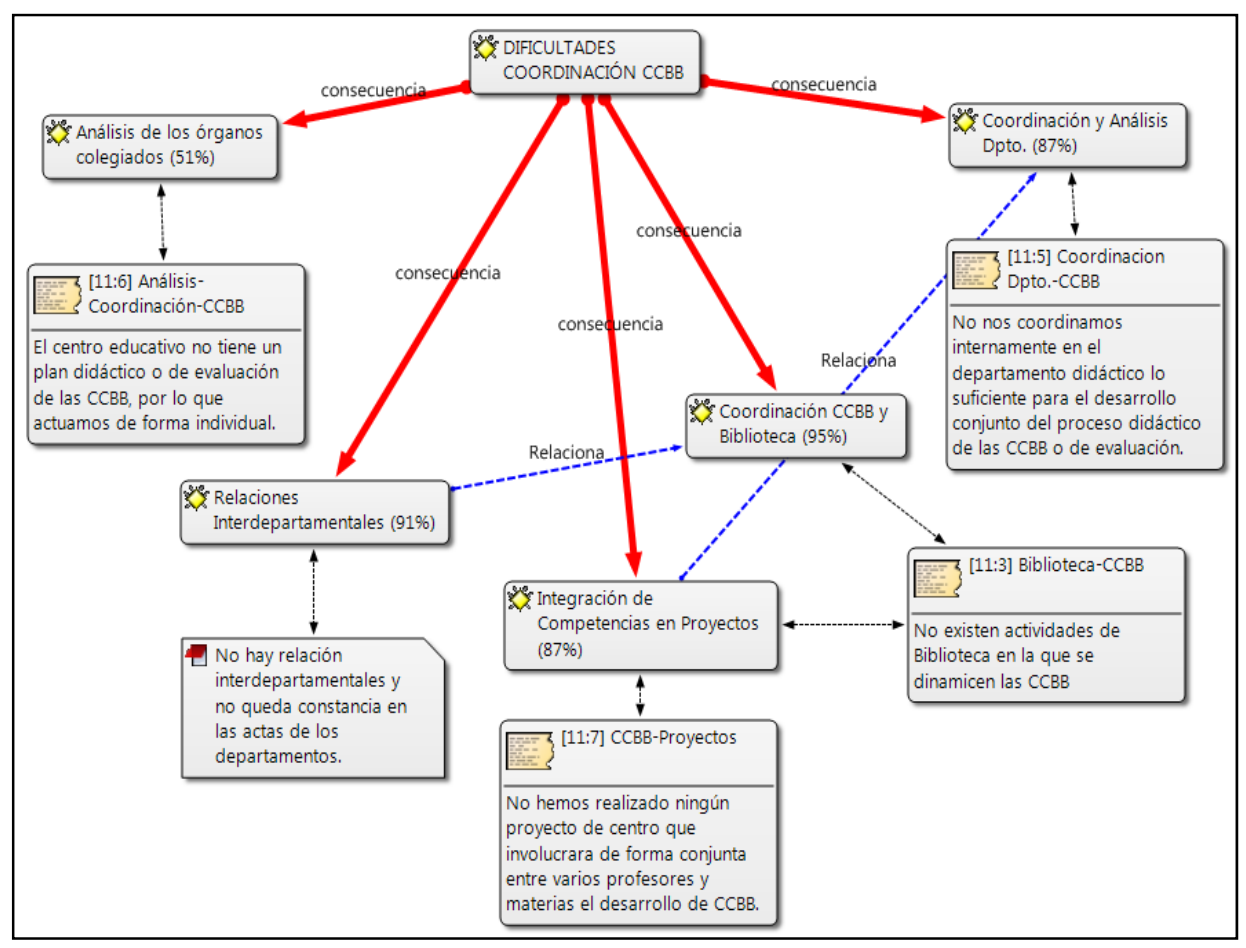

Figura 2. Dificultades del proceso de coordinación de las competencias claves.

El proceso de coordinación para la programación y evaluación de las competencias claves presenta dificultades que obstaculizan gravemente su desarrollo y evaluación. Una de las dificultades más acusadas es la falta de coordinación interna en los departamentos didácticos y equipos de ciclo. Resulta esencial fomentar procesos fuertes de coordinación interna en estos órganos, ya que el desarrollo metodológico y 
evaluativo, parte de un trabajo del profesorado colaborativo y contextualizado en su materia.

\section{Dimensión 3: Dificultades en la didáctica y evaluación de las competencias claves.}

Esta dimensión afecta directamente al trabajo del docente en sus funciones de enseñar y evaluar recogidas como funciones prioritarias en el artículo 91 de la LOMCE (2013) y que parte de un buen diseño de las otras dos dimensiones analizadas: planificación y coordinación. El proceso de diseño de actividades, tareas o proyectos relacionados con los contenidos de la materia que imparte cada docente $\mathrm{y}$, de forma interdisciplinar con la de otros docentes, constituye una dimensión prioritaria y necesaria en el desarrollo y evaluación de las competencias. El profesorado -a priori- tiene problemas para identificar contenidos susceptibles de ser transformados en competencias a través de actividades o tareas que impliquen la aplicación de esa habilidad o destreza en un contexto social, académico o personal del alumnado. Asimismo, se detectan problemas a la hora de anotar, calificar y evaluar esas actividades o tareas y relacionarlas con los criterios de evaluación. Por último, no suele haber análisis rigurosos del desarrollo de estas competencias o procesos de autoevaluación que puedan servir de referente en la mejora del proceso de enseñanzaaprendizaje. En la Tabla 8, se presentan las frecuencias de significado de las categorías más determinantes para entender las dificultades para afrontar la didáctica y evaluación de las competencias claves desde una perspectiva individualizadora de la actuación docente.

\begin{tabular}{|c|c|c|c|c|}
\hline $\begin{array}{l}\text { Dominio: Dificultad Didáctica y Evaluación de } \\
\text { Competencias }\end{array}$ & Casos & $\%$ Casos & Nb Words & \% Words \\
\hline Estrategias didácticas. & 111 & $8,7 \%$ & 211 & $10,4 \%$ \\
\hline Relación contenidos y competencias. & 123 & $9,6 \%$ & 232 & $11,5 \%$ \\
\hline Anotación del progreso de las competencias. & 213 & $16,7 \%$ & 341 & $16,9 \%$ \\
\hline Evaluación y calificación de las competencias. & 187 & $14,7 \%$ & 321 & $15,9 \%$ \\
\hline Planes de formación. & 401 & $31,5 \%$ & 532 & $26,4 \%$ \\
\hline Uso de las TIC como eje transversal. & 234 & $18,4 \%$ & 377 & $18,7 \%$ \\
\hline Total & 1269 & 100 & 2014 & 100 \\
\hline
\end{tabular}

Tabla 8. Dificultades en metodología y de evaluación de competencias.

Las frecuencias más determinantes que explican la dificultad para programar y evaluar competencias por parte del profesorado se centran principalmente en: "la ausencia de planes de formación" (Casos=401/Nb-Words: 532) y "la anotación del progreso de las competencias en los diarios del profesorado conforme a los criterios de evaluación" (Casos=400/Nb-Words: 563). En la Figura 3, de forma más visual, se muestran las principales dificultades en el proceso de didáctica y evaluación de las competencias claves en porcentajes de aparición en Atlas-Ti. 


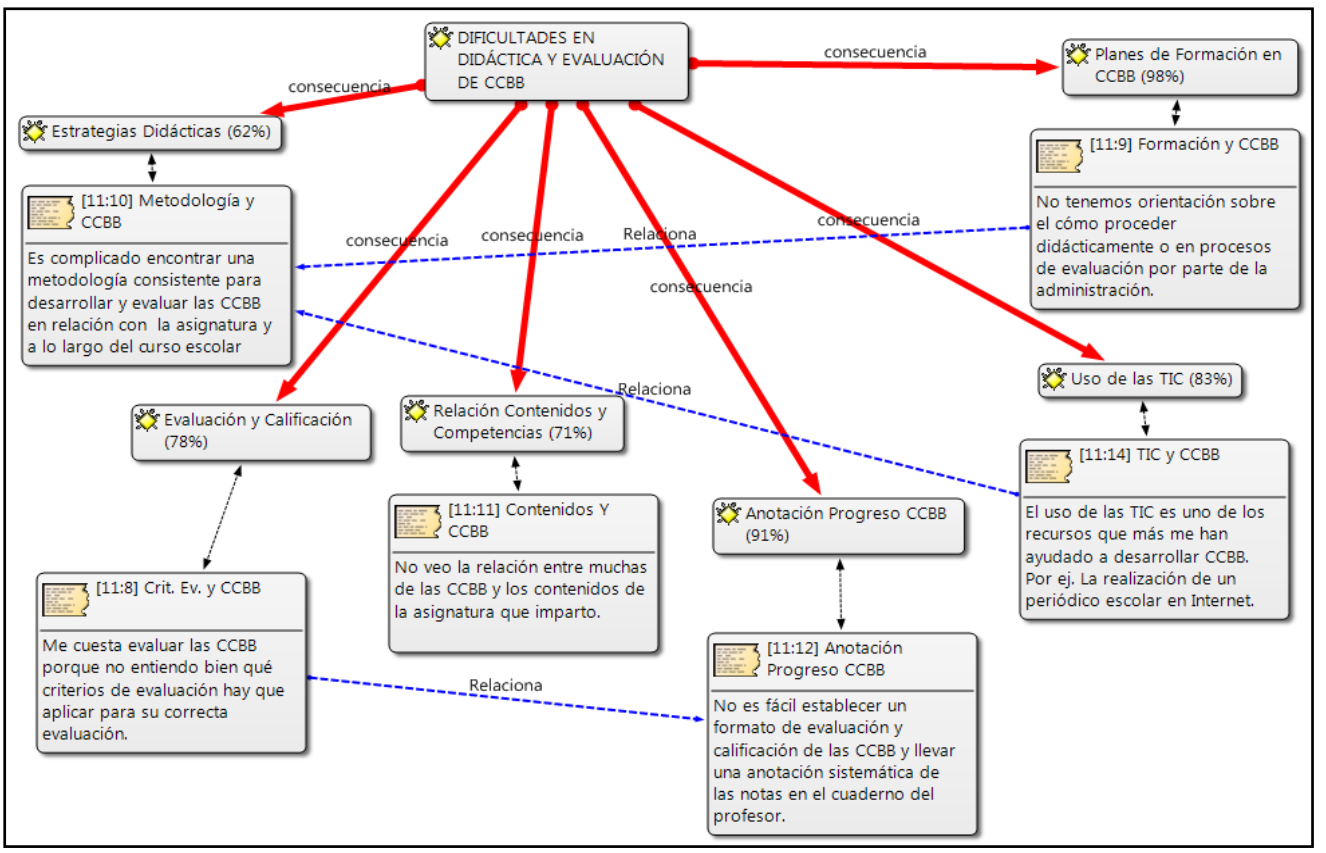

Figura 3. Dificultades para la didáctica y de evaluación de las competencias claves.

Las principales dificultades observadas en los procesos metodológicos y evaluadores de las competencias claves en los centros educativos se concretan en los siguientes ámbitos:

- Un $87 \%$ del profesorado $\mathrm{N}=455$ no ha realizado ningún curso de formación específico en los ámbitos de metodología y evaluación de competencias.

- Un 91\% del profesorado $\mathrm{N}=476$ encuentra difícil la anotación en su diario de clase de la nota obtenida en las tareas por competencias según indicadores de evaluación del decreto de currículo.

- Un $62 \%$ del profesorado $\mathrm{N}=324$ considera complicado encontrar contenidos relacionados con competencias.

- Un 95,7\% del profesorado $\mathrm{N}=501$ considera complicado desarrollar estrategias didácticas para la impartición continuada de competencias.

Las principales dificultades mostradas por el profesorado se centran principalmente en la falta de planes de formación para un desarrollo efectivo de las competencias y de los procesos de evaluación. Este aspecto es crucial para una buena consecución del proceso metodológico y evaluador de las competencias. Relacionado con este aspecto 
es de reseñar que un $83 \%$ del profesorado $\mathrm{N}=434$ percibe las TIC como una de las mejores estrategias para desarrollar competencias en el aula y de forma colaborativa.

\section{Análisis de redes sociales aplicado a los grupos de discusión}

El estudio de frecuencias textuales realizado con el programa Atlas-Ti se complementó con el análisis de las percepciones, comentarios y opiniones de los diferentes miembros de la comunidad educativa en 5 grupos de discusión realizados en los centros educativos con la participación de dos miembros representantes de cada sector de la comunidad educativa: equipo directivo, profesor/a, padre/madre, alumno/a desde el enfoque metodológico del análisis de redes sociales (ARS). Analizamos la red de interacciones obtenida en la transcripción escrita del archivo sonoro de cada grupo de discusión. Para ello, hemos editado la red resultante de UCINET con el editor yED Graph Editor 3.11.1 (Figura 4) para hacerla más visual y comprensible.

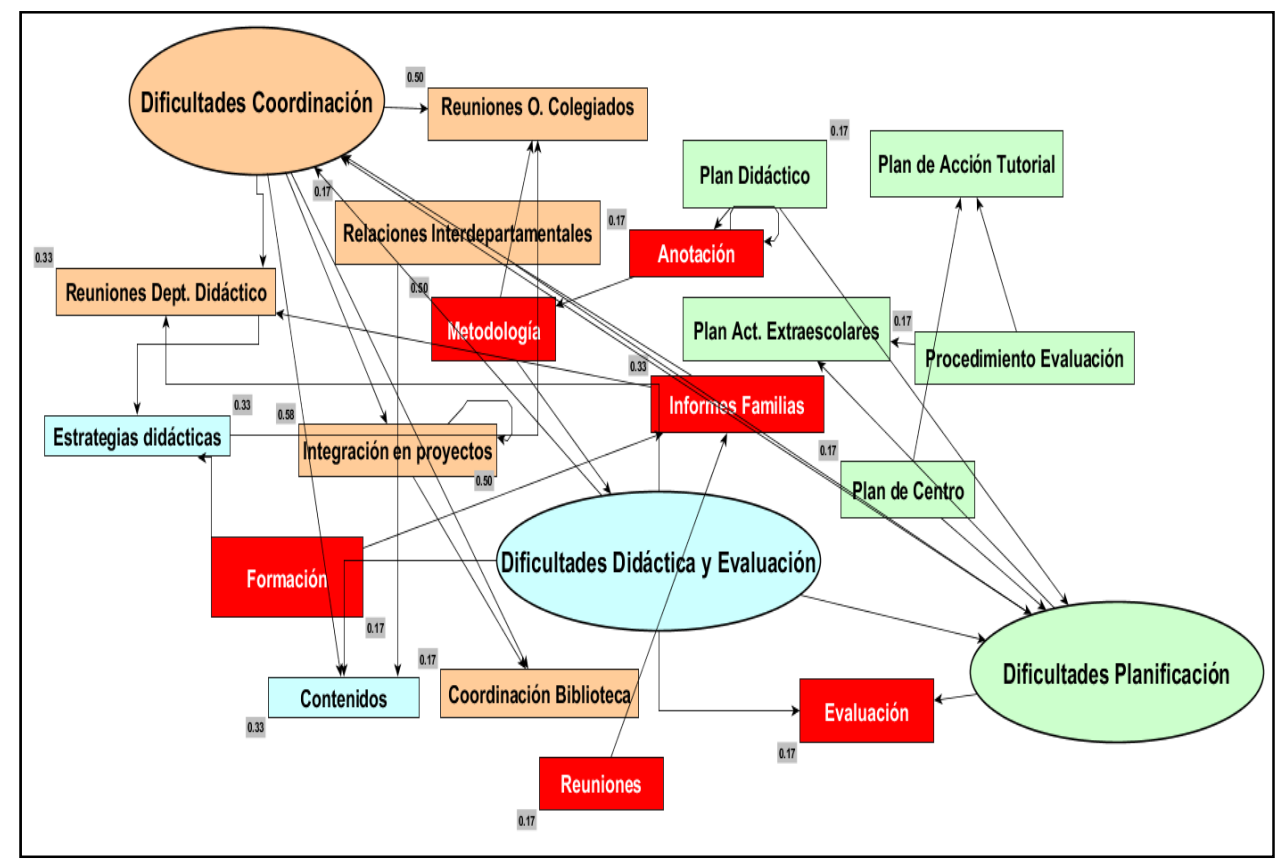

Figura 4. Red de competencias

Podemos observar tres nodos principales en formato elipse y tres colores para facilitar su identificación en la red. La densidad media de los cinco grupos de discusión con la matriz dicotomizada fue .81 con .24 de desviación estándar; lo que representa un valor alto y un rango promedio de la red de 2.610; lo cual indica que cada palabra clave está interrelacionada con una media de casi 3 . Este valor se considera alto para un total 
de 15 nodos centrales. Además, este resultado muestra que más de dos tercios de todos los vínculos posibles estuvieron presentes y que se obtuvo una alta participación. Hemos analizado la centralidad de la red para identificar aquellos aspectos más prominentes. Para ello, hemos recurrido al análisis del grado nodal, de intermediación y de cercanía (Tabla 9).

\begin{tabular}{|l|c|c|c|c|c|c|}
\hline \multicolumn{1}{|c|}{ Ítems } & \multicolumn{2}{c|}{$\begin{array}{c}\text { Grado } \\
\text { Nodal }\end{array}$} & \multicolumn{2}{c|}{$\begin{array}{c}\text { Grado } \\
\text { Intermediación }\end{array}$} & \multicolumn{2}{c|}{$\begin{array}{c}\text { Grado } \\
\text { Cercanía }\end{array}$} \\
\hline \multicolumn{1}{|c|}{ Dificultades Planificación } & Deg. & NrmD. & Bet. & nBet. & Far. & Clos. \\
\hline 1.Plan de Centro & 23.0 & 65.120 & 85.0 & 42.0 & 71.0 & 39.21 \\
\hline 2.Informe a Familias & 25.0 & 72.175 & 71.5 & 31.0 & 73.0 & 38.11 \\
\hline 3.Procesos de evaluación & 24.0 & 68.150 & 63.5 & 55.0 & 70.0 & 36.45 \\
\hline 4.Plan de A. Tutorial & 21.0 & 58.750 & 75.0 & 31.5 & 67.5 & 31.51 \\
\hline 5. Pan Actv. Extraescolares & 20.0 & 60.225 & 72.0 & 30.5 & 65.5 & 32.00 \\
\hline 6. Plan Didáctico & 22.0 & 60.750 & 82.5 & 30.5 & 61.5 & 33.00 \\
\hline \multicolumn{1}{|c|}{ Dificultades coordinación } & Deg. & NimD. & Bet. & nBet. & Far. & Clos. \\
\hline 7. Reuniones órganos colegiados & 15.0 & 45.500 & 40.0 & 22.0 & 51.5 & 33.00 \\
\hline 8. Reuniones dpto. didácticos & 23.5 & 59.125 & 55.5 & 30.0 & 61.0 & 41.10 \\
\hline 9. Relaciones interdepartamentales & 22.0 & 61.450 & 59.5 & 35.0 & 70.0 & 46.50 \\
\hline 10.Integración en proyectos & 23.0 & 68.150 & 65.5 & 37.5 & 69.5 & 41.50 \\
\hline 11.Coordinación Biblioteca & 23.0 & 65.155 & 62.5 & 38.5 & 68.5 & 45.00 \\
\hline Dificultades Did. y Evaluación & Deg. & NimD. & Bet. & nBet. & Far. & Clos. \\
\hline 12.Formación & 23.5 & 60.120 & 55.5 & 30.0 & 61.0 & 41.10 \\
\hline 13.Anotación & 23.0 & 62.455 & 59.5 & 35.0 & 70.0 & 46.50 \\
\hline 14.Contenidos adecuados & 16.0 & 47.155 & 65.5 & 37.5 & 69.5 & 41.50 \\
\hline 15.Estrategias didácticas & 24.0 & 65.450 & 62.5 & 38.5 & 68.5 & 45.00 \\
\hline Nodos centrales & Deg. & NimD. & Bet. & nBet. & Far. & Clos. \\
\hline 16.Formación & 23.5 & 60.120 & 55.5 & 30.0 & 61.0 & 41.10 \\
\hline 17. Procesos de Evaluación & 24.0 & 68.150 & 63.5 & 55.0 & 70.0 & 36.45 \\
\hline 18.Reuniones dpto. didácticos & 23.5 & 59.125 & 55.5 & 30.0 & 61.0 & 41.10 \\
\hline 19.Informe a Familias & 25.0 & 72.175 & 71.5 & 31.0 & 73.0 & 38.11 \\
\hline 20.Metodología & 24.0 & 65.450 & 62.5 & 38.5 & 68.5 & 45.00 \\
\hline 21.Anotación & 23.0 & 62.455 & 59.5 & 35.0 & 70.0 & 46.50 \\
\hline
\end{tabular}

Tabla 9. Grado nodal, de intermediación y cercanía en la red "Competencias"

La centralidad muestra la posición de los conceptos en la red (Spencer, 2003) y arroja un resultado bastante alto del $71 \%$ con un número total de nodos de 15 . El grado máximo (número máximo de relaciones de un nodo en la red) es de 25 ("Informe a Familias"). El resultado del grado de intermediación en la red 59,002, lo que nos proporciona información relevante con respecto a la frecuencia con que aparece un nodo en el tramo más corto (o geodésico) que conecta a otros dos; es decir, muestra cuando un tema puede ser intermediario entre otros. Hemos reseñado en el grupo "Nodos centrales" aquellos nodos que tienen un grado de intermediación mayor $(\geq 20)$ y son recurrentes en las tres dominios analizados y a los que podemos denominar "núcleos de centralidad del grafo" según el concepto de "k-cores" (Seidman, 1983) y que se concentran en los siguientes: "formación" (23,5), "procesos de evaluación" (24), "reuniones de departamentos didácticos" (23,5), "metodología (24) y "anotación" (23). 


\section{Conclusiones}

La investigación muestra cómo las dificultades del profesorado para integrar y evaluar las competencias claves en los procesos de enseñanza-aprendizaje presentan deficiencias en las tres grandes categorías analizadas: planificación, coordinación y procesos didácticos y evaluadores. Los procesos de planificación, coordinación, programación y evaluación de las competencias son manifiestamente mejorables en la mayoría de los centros educativos analizados, lo que puede llegar a conculcar uno de los derechos fundamentales del alumnado a una evaluación objetiva (LODE: Art.6b y RD 732/1995 Art. 13.1).

Las dificultades del proceso de planificación de competencias muestran la falta de planes de centro consensuados y la casi inexistencia de planificación de actividades tutoriales y actividades extraescolares que potencien el desarrollo de las competencias en los centros educativos. Asimismo, no se informa periódicamente del desarrollo y adquisición de las competencias al alumnado y a sus familias. Este proceso se extiende a lo largo de toda la enseñanza obligatoria (Primaria y Secundaria), por lo que un alumno/a debería disponer de un informe de progreso de competencias claves desde primero de enseñanza primaria hasta su titulación en cuarto de la ESO; informe que debería ser notificado a las familias durante cada curso académico y al finalizar cada curso escolar. La realidad muestra que estos informes raramente forman parte de los documentos informativos de los centros y de la práctica diaria del profesorado. Estos resultados confirman también dificultades encontradas en otros trabajos sobre el papel de la administración educativa en el planteamiento de las competencias claves (Roselló y Pinya, 2014).

Junto con las dificultades de planificación, los procesos de coordinación de las competencias adolecen de falta de colaboración y consenso. De esta forma, no hay análisis rigurosos de los órganos colegiados y departamentos didácticos que ayuden a solventar los problemas de impartición y evaluación de las competencias. Existe poco trabajo colaborativo interdepartamental e interdisciplinar; lo que confiere a los centros unas estructuras rígidas poco proclives al desarrollo de proyectos con base en las competencias. Esta situación agudiza la dificultad del profesorado para relacionar competencias con contenidos y establecer estrategias metodológicas para su desarrollo. Asimismo, la evaluación y anotación del progreso en las competencias es una faceta muy poco trabajada en los diarios de clase del profesorado. Es destacable, también, la disconformidad del profesorado con la formación recibida en competencias tanto en aspectos didácticos como evaluadores. Un alto porcentaje de los mismos manifiesta que las competencias aparecieron en la normativa pero que no ha llegado su desarrollo efectivo a las aulas por falta de formación para su desarrollo.

A la vista de los resultados de esta investigación, parece necesario mejorar sustancialmente los procesos de planificación, coordinación y evaluación de las competencias claves como referente fundamental para el alumnado a la hora de promocionar de curso y obtener el título de Graduado en Secundaria, y con ello, dar cumplimiento a la normativa vigente. El profesorado debe reflexionar y orientar la práctica docente y evaluativa de las competencias claves a una revisión que responda a las siguientes preguntas: 
- ¿Se imparten actividades basadas en competencias básicas y se relacionan con los bloques de contenido del currículo?

- ¿Se desarrollan durante un curso académico actividades, tareas o proyectos relacionados con las diferentes competencias básicas?

- ¿Se aplican variados procedimientos de evaluación para evaluar competencias básicas? O se suele evaluar con exámenes.

- ¿Se evalúan de forma efectiva y continuada las competencias básicas y, para ello, se utilizan los criterios de evaluación del decreto de currículo correspondiente?

- ¿Se informa adecuadamente y de forma completa a alumnado y familias sobre el proceso de adquisición de las diferentes competencias básicas; orientando eficazmente su mejora?

- ¿Se trabaja de forma conjunta y colaborativa en un plan de centro para el desarrollo y evaluación de las competencias básicas conforme a los documentos programáticos del centro educativo?

Las respuestas a las anteriores preguntas deben servir para reflexionar sobre la posibilidad de impartir clase atendiendo a los contenidos de los decretos de currículo en toda su variedad y aplicar variados procedimientos de evaluación que reflejen los diferentes estilos de aprendizaje; [no sólo centrados en comprobar la memorización de determinados contenidos y enfocar la práctica educativa hacia una vertiente más práctica y funcional (competencia)].

Por último, es reseñable que el desarrollo de las competencias claves en el alumnado es el referente mundial de las pruebas PISA, en las que España no está obteniendo buenos resultados en los últimos años; sería, por lo tanto, deseable ver cómo se están planificando, coordinando, impartiendo y evaluando las competencias en otros países y de forma comparada entre comunidades autónomas en España.

\section{Referencias bibliográficas}

BARABÁSI, A. L. (2002). Linked - The New Science of Networks. Cambridge: Perseus Publishing.

BRENNAN, R. L., \& PREDIGER, D. J. (1981). Coefficient Kappa: Some Uses, Misuses, and Alternatives. Educational and Psychological Measurement, 41, 68799.

BOLÍVAR, A. (2008). Ciudadanía y competencias básicas. Sevilla: Fundación ECOEM.

BRETT, J. M., SHAPIRO, D. L., \& LYTLE, A. L. (1998). Breaking the Bonds of Reciprocity in Negotiations. Academy of Management Journal, 41, 410-24.

BROWNELL, J. E., \& SWANER, L. E. (2010). Five high-impact practices: Research on learning outcomes, completion, and quality. Washington, D.C.: Association of American Colleges and Universities. 
CASANOVA, M. A. (2004). Evaluación y calidad de centros educativos. Madrid: La Muralla.

CASANOVA, M. A. (2012). La evaluación de competencias básicas. Madrid: La Muralla.

CASTELLS, M., \& MONGE, P. (2011). Network Multidimensionality in the Digital Age. International Journal of Communication, 5, 788-793.

CAVERLEE, J., LIU, L., \& WEBB, S. (2010). The Social Trust framework for trusted social information management: Architecture and algorithms. Information Sciences, ScienceDirect, 180, 95-112.

CHARMAZ, K. (2001). Qualitative interviewing and grounded theory analysis. En J.F. Gubrium y J.A. Holstein Handbook of interview research, London: Sage.

CLANDININ, J., \& CONNELLY, M. (2000). Narrative inquiry: experience and story in qualitative research. San Francisco, CA: Jossey Bass.

DARESH, J. C., \& PLAYKO, M. A. (1995). Supervision as a Proactive Process. Prospect Heights: Waveland Press.

DEAN, J. (1997). Supervisión y asesoramiento. Manual para inspectores, asesores y profesorado asesor. Madrid: La Muralla.

DIARIO OFICIAL DE LA UNIÓN EUROPEA (2006). Recomendación del parlamento europeo y del consejo de 18 de diciembre de 2006 sobre las competencias clave para el aprendizaje permanente. L 394/10. (2006/962/CE) 30 de diciembre de 2006.

ESCAMILLA, A. (2008). Las competencias básicas. Claves y propuestas para su desarrollo en los centros. Barcelona: Graó.

EUROPEAN COMMISSION (2007). Key competences for lifelong learning. European Reference Framework. Luxembourg: Office for Official Publications of the European Communities.

FLEISCHMAN, H. L., HOPSTOCK, P. J., PELCZAR, M. P., \& SHELlEY, B. E. (2010). Highlights From PISA 2009: Performance of U.S. 15-Year-Old Students in Reading, Mathematics, and Science Literacy in an International Context (NCES 2011-004). Washington, DC: National Center for Education Statistics, Institute of Education Sciences, U.S. Department of Education.

GIMENO-SACRISTÁN, J. (Compl.) (2008). Educar por competencias ¿Qué hay de nuevo? Madrid: Morata.

HOLSTI, O. R. (1969). Content Analysis for the Social Sciences and Humanities. Reading, MA: Addison-Wesley.

KNOKE, D., \& Yang, S. (2008). Social Network Analysis. USA: SAGE.

LEY ORGÁNICA 2/2006, de 3 de mayo, de Educación.

LEY ORGÁNICA 8/2013, de 9 de diciembre, para la mejora de la calidad educativa. 
LEY ORGÁNICA 8/1985, de 3 de julio, reguladora del Derecho a la Educación.

LOMBARD, M., SNYDER-DUCH, J., \& BRACKEN, C. (2002). Content Analysis in Mass Communication: Assessment and Reporting of Intecoder Reliability. Journal of International Business Studies, 36, 357-78.

MEC (2013). PISA 2012. Informe Español. Volumen I: Resultados y contexto. Madrid: Instituto Nacional de Evaluación Educativa.

OECD (2009). PISA 2009 assessment framework: Key competencies in reading, mathematics and science literacy. OECD Publishing.

OECD (2011). PISA 2009 results: Students on line-Digital technologies and performance (Volume VI). OECD Publishing.

OCDE DeSeCo. Definition and Selection of Competencies: Theoretical and Conceptual Foundations. http://www.oecd.org/fr/edu/apprendre-au-dela-de-1ecole/definitionandselectionofcompetenciesdeseco.htm (Consultado 1 de octubre de 2014).

PROYECTO ATLÁNTIDA (2014). De las competencias básicas al currículo integrado. Madrid: Proyecto Atlántida.

REAL DECRETO 732/1995, de 5 de mayo, por el que se establecen los derechos y deberes de los alumnos y las normas de convivencia en los centros.

REAL DECRETO 126/2014, de 28 de febrero, por el que se establece el currículo básico de la Educación Primaria.

ROSELLÓ, M. ${ }^{a}$ R. Y PINYA, C. (2014). La formación en competencias básicas: un reto para la administración. Profesorado, 18(2), 245-265.

SANMARTÍ, N. (2007). 10 ideas clave: evaluar para aprender. Barcelona. GRAÓ.

SEIDMAN, S.B. (1983). Network structure and minimum degree. Social Network, 5, 269-287.

SIMONS, T. (1993). Speech Patterns and the Concept of Utility in Cognitive Maps: The Case of Integrative Bargaining. Academy of Management Journal, 36, 139-56.

SPENCER, J. W. (2003). Global gatekeeping, representation and network structure: a longitudinal analysis of regional and global knowledge-diffusion networks. Journal of international business studies, 34, 428-442.

STRAUSS, A., \& Corbin, J. (1997). Grounded theory in practice. London: Sage.

VÁZQUEZ-CANO, E., SEVILLANO, M.a L., \& MÉNDEZ, M. (2011). Programar en Primaria y Secundaria. Madrid: Pearson.

VÁZQUEZ-CANO, E. (2012a). La evaluación del aprendizaje en primaria y secundaria: los indicadores de evaluación. Espiral, Cuadernos del profesorado, 5(10), 30-41. 
VÁZQUEZ-CANO, E. (2012b). Propuesta y análisis de un modelo para la supervisión e inspección de los procesos de evaluación del alumnado en centros educativos "MOSUPEV": un estudio de casos. Revista Iberoamericana sobre Calidad, Eficacia y Cambio en Educación (REICE), 10(4), 115-133.

WEINGART, L.R., OLEKALNS, M., \& SMITH P. (2004). Quantitative Coding of Negotiation Behavior. International Negotiation, 9, 441-55.

\section{Correspondencia con el autor}

Esteban VÁZQUEZ-CANO

Universidad Nacional de Educación a Distancia

Facultad de Educación

Departamento de Didáctica, Organización Escolar y Didácticas Especiales

C/ Juan del Rosal, 14 Madrid (28040)

evazquez@edu.uned.es 